\title{
Hacia el desarrollo de una aplicación colaborativa para el diseño de diagramas de clases en ambientes educativos
}

\author{
S. David Díaz-García ${ }^{1}$, Luis G. Montané-Jiménez ${ }^{2}$, Betania Hernández-Ocaña ${ }^{3}$ \\ 1 Laboratorio Nacional de Informática Avanzada, \\ Xalapa, México \\ 2 Universidad Veracruzana, \\ Facultad de Estadística e Informática, Xalapa, México \\ 3 Universidad Juárez Autónoma de Tabasco, \\ Villahermosa, Tabasco, México
}

sdiaz.mca15@lania.edu.mx, lmontane@uv.mx, betania.hernandez@ujat.mx

\begin{abstract}
Resumen. Cada vez más compañías utilizan tecnologías colaborativas para apoyar el trabajo de equipo mediante computadoras, mejorando la comunicación e interacción de manera centralizada o distribuida. En el trabajo de equipo, tener fácil acceso a información del resto del equipo, puede determinar el cumplimiento exitoso de metas. En el trabajo colaborativo, un elemento de diseño que permite compartir información es el awareness o consciencia de grupo. Con awareness es posible compartir información que ayude a entender cómo se desarrolla la actividad de equipo. En actividades colaborativas desarrolladas en ambientes educativos tradicionales, por ejemplo, diseño de diagramas de clases en un pizarrón, se dificulta identificar tareas realizadas y el grado de contribución de los miembros de equipo. Este trabajo presenta de manera conceptual una arquitectura funcional y el diseño de una aplicación colaborativa para diseñar diagramas de clases en un ambiente educativo, contemplando el uso de agentes para el mejoramiento del awareness.
\end{abstract}

Palabras clave: consciencia, trabajo colaborativo asistido por computadora, groupware, agentes.

\section{Towards the Development of a Collaborative Application for Design of Class Diagrams in Educational Environments}

\begin{abstract}
Nowadays, the companies use collaborative technologies to support teamwork through computers, improving communication and interaction in a centralized or distributed way. In teamwork, having easy access to information from the rest of the team, can determine the
\end{abstract}


successful accomplishment of goals. In collaborative work, a mechanism for sharing information is awareness. With awareness, it is possible to share information that helps to understand how the team activity is developed. In collaborative activities developed in traditional educational environments, e.g., designing class diagrams on a blackboard, it is difficult to identify tasks performed and the degree of contribution of team members. Therefore, in this paper we present a functional architecture and the design of a collaborative application in order to design class diagrams in an educational environment, contemplating the use of agents for the improvement of awareness.

Keywords: awareness, computer-supported cooperative work, groupware, agents.

\section{Introducción}

El trabajo colaborativo asistido por computadora (Computer-Supported Cooperative Work) es una área que se enfoca en estudiar ambientes donde personas que trabajan de forma conjunta son apoyadas con tecnología computacional, con la finalidad de desarrollar una actividad de equipo que define metas y objetivos comunes [1]. Los sitemas que apoyan el desarrollo de actividades colaborativas son denominados sistemas Groupware o Colaborativos. En la mayoría del trabajo colaborativo se requiere de las personas un grado de comunicación y cooperación con los demás, a eso se le llama trabajo de equipo. Sin embargo, todavía se han observado dificultades de coordinación y comunicación en equipos y organizaciones [2]. Estas dificultades tienden a contrarrestar los beneficios del trabajo colaborativo y la toma de decisiones [3]. En ambientes tradicionales educativos, como un escenario llevado a aulas de clases, un maestro solicita a sus alumnos realizar una tarea de equipo en un pizarrón, por ejemplo, diseñar un diagrama de clases. Realizar este tipo de tareas en un pizarrón tradicional, es decir, que no es apoyado por un sistema de información, dificulta a los participantes y al evaluador identificar la participación de todos los integrantes de equipo, además de impedir trabajar de manera fluida al no poder escribir en el pizarrón todos los participantes al mismo tiempo debido al espacio de trabajo. Para apoyar la identificación de estos niveles de contribución y ayudar en la organización de tareas de equipo en el espacio de trabajo se puede hacer uso de agentes $[4,5,6]$.

Con la finalidad de mejorar el trabajo de equipo para la actividad mencionada y ayudar a formar una opinión social más informada de los participantes durante la actividad, se propone el desarrollo de una aplicación colaborativa para el diseño de diagramas de clases en ambientes educativos, que pueda ser implementado posteriormente en ambientes de escritorio, y finalmente adaptado con pizarras interactivas. Por lo tanto, esta propuesta inicial de aplicación permitiría trasladar una actividad educativa desarrollada en ambientes tradicionales a un ambiente digital a través de un sistema Groupware. El uso de este Groupware mantendría un contexto y consciencia sobre lo realizado por cada uno de los miembros de un equipo. 
Hacia el desarrollo de una aplicación colaborativa para el diseño de diagramas de clases ...

Este documento está estructurado de la siguiente forma: la Sección 2 presenta sistemas Groupware, awareness y sus aplicaciones, la Sección 3 describe el diseño de la aplicación colaborativa propuesta. Finalmente, la Sección 4 presenta las conclusiones y trabajo futuro.

\section{Sistemas groupware y awareness}

\subsection{Sistemas groupware}

Los requerimientos y problemas que enfrentan las empresas demandan esfuerzos organizados de equipo [7], para facilitar cumplir con estos requerimientos y solucionar los problemas existen los sistemas Groupware. En [8] Groupware se define como un sistema basado en computadoras que apoya el trabajo de equipo de personas que trabajan en una tarea o meta común. Los sistemas Groupware permiten a un equipo trabajar en una problemática uniendo esfuerzos apoyados por software.

Algunas áreas de aplicación de los sistemas Groupware son los ambientes de conocimiento colaborativo (p.ej. las Wikis [9]), en entretenimiento están los videojuegos online como AssaultCube [10], herramientas de desarrollo de software como CALICO [11], es un Groupware basado en pizarras interactivas que permiten diseñar sketches en un medio fluido, flexible y colaborativo. CALICO permite trabajar sketches de manera colaborativa síncrona y asíncrona, retomar trabajo, trabajar con múltiples lienzos y navegar entre ellos. El problema con estas aplicaciones es que es difuso identificar la aportación de trabajo de los participantes de equipo, además de carecer de un modelo de actividad que permita modelar los elementos que intervienen en la actividad colaborativa ( $p$. ej. tareas, actores y la meta del equipo) para ser contabilizados.

\subsection{Escenarios de aplicación}

Durante el trabajo colaborativo en las interfaces de usuario pueden ocurrir 3 tipos de escenarios [12]: i) Misma tarea - Misma vista. Este escenario ocurre cuando dos o más colaboradores trabajan en la misma actividad al mismo tiempo, ellos ven los mismo. ii) Misma tarea - Diferente vista. Sucede cuando dos o más colaboradores realizan una tarea que involucra acciones coordinadas en diferentes áreas de trabajo. iii) Misma tarea - Enfoque Mixto. Involucra actividades individuales y de equipo, y los usuarios desplazan su atención entre vistas separadas y compartidas en el espacio de trabajo.

En el trabajo colaborativo el conocimiento compartido ayuda a mejorar el trabajo de equipo [13]. Los usuarios que colaboran en equipo requieren conocer su situación de trabajo y la de sus compañeros con el fin de completar sus actividades satisfactoriamente. Por medio de awareness podemos representar información de la actividad del equipo, de lo que ocurre en el sistema, y compartirla entre el equipo. 


\subsection{Awareness y sus aplicaciones}

Para [14] "awareness es información que el sistema provee sobre el estado de la colaboración en relación a la presencia de los usuarios, su ubicación dentro del sistema, nivel de actividad, acciones, cambios, objetos que se utilizan, intención etc.". Mientras que en [15] los autores lo definen como "la percepción de los elementos del sistema con respecto al espacio y tiempo.". Las dos definiciones plantean conceptos importantes, en este documento awareness está basado en la primera definición, y donde se propone un mecanismo para intercambiar información que proporcione una idea del estado de actividad de los demás compañeros de equipo y del sistema en términos de desempeño.

Usar awareness permite informar al equipo de la actividad que sus integrantes realizan y lo que debería hacer el equipo. Esto proporciona beneficios como mejorar la coordinación sin necesidad de comunicarse abiertamente, capacidad para pronosticar el resultado del curso de una acción, y también proporciona un entendimiento de contexto [16].

Utilizar awareness tiene aplicaciones en diversas áreas, p. ej. la salud, el entretenimiento o el conocimiento compartido. Un ejemplo de awareness se presenta en la Figura 1, esta vez en el área del entretenimiento (videojuegos online), en la Figura 1 (a) [17] el awareness muestra los compañeros y enemigos que pueden continuar jugando en la partida. En [18] se presenta un juego serio llamado "Escape from Wilson Island", es sobre un juego colaborativo que incluye habilidades sociales como trabajo de equipo, coordinación y comunicación. En la interfaz de usuario se muestran elementos como chat, un minimapa, y atributos relevantes de los demás compañeros (ver Figura 1 (b)).

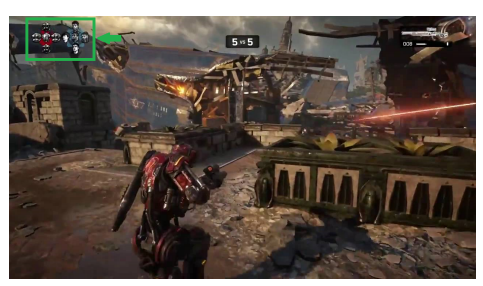

(a) Gears of war

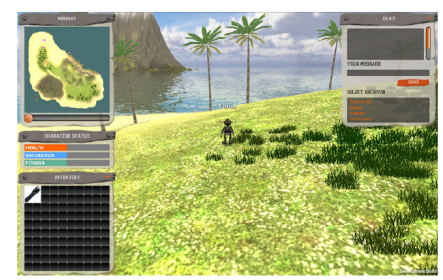

(b) Escape from Wilson Island

Fig. 1. Ejemplo de awareness en el entretenimiento (videojuegos online).

Tele-Board [19] es un sistema colaborativo que utiliza pizarras digitales basadas en Web. En Tele-Board, el awareness son notas adhesivas que sirven como medio para comunicarse entre el equipo de trabajo (ver Figura 2). 
Hacia el desarrollo de una aplicación colaborativa para el diseño de diagramas de clases ...

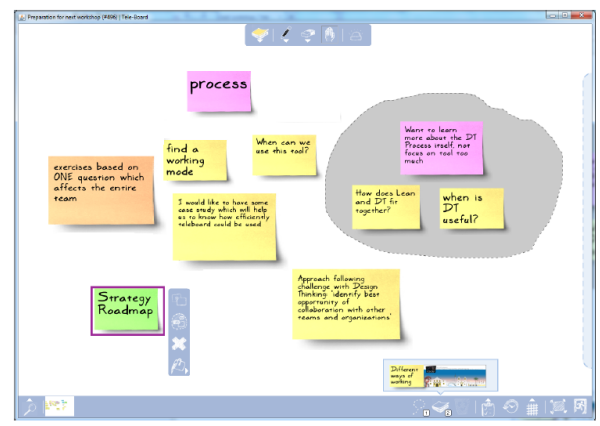

Fig. 2. Notas adhesivas que se utilizan como awareness en Tele-Board.

Para mejorar la interactividad de los Groupware, se plantea el uso de mecanismos de razonamiento que utilicen información de awareness para generar comportamientos reactivos dependiendo el estado de una actividad o de los usuarios. Esto apoyaría la creación de sistemas Groupware de siguiente generación.

\subsection{Tipos de awareness}

Los sistemas Groupware ofrecen mecanismos de awareness, basados en tiempo, lugar y espacio se conocen seis categorías de awareness en [20]: de colaboración, de ubicación, de contexto, sociales, de espacio de trabajo o de situación. De colaboración se refiere a la manera en la cual miembros de un equipo interactúan: síncronamente, asíncronamente o una mezcla de ambas. Un awareness de ubicación está basado en las relaciones geográficas entre colaboradores como ubicación, distancia, orientación y rango de atención. Por otra parte, awareness de contexto es fundamental para permitir a un equipo de colaboradores mantener un sentido de qué es lo está sucediendo en el espacio virtual. Social awareness comprende los roles y actividades de los demás miembros de equipo y como los miembros están contribuyendo a las tareas. Mientras de espacio de trabajo se enfatiza en un lugar que sirve para organizar tareas de acuerdo a un conjunto lógico por ejemplo un editor de equipo que sirve para organizar tareas como escribir y revisar mientras se mantiene el trabajo en la actividad que se desarrolla. Finalmente, un awareness de situación emerge de los esfuerzos individuales, de coordinación y colaboración. Esta clasificación es centrada en compartir información que permita conocer lo que ocurre en el ambiente de trabajo p. ej. las actividades que se realizan, el tipo de colaboración (centralizado o distribuido) y los objetos que intervienen.

En [12] se propone clasificar awareness en aspectos: i) sociales, contempla si una persona presta atención, su estado emocional y nivel de interés, ii) de tareas, considera el conocimiento para contemplar una tarea, herramientas necesarias, tiempo necesario y disponible, iii) de conceptos, atiende cómo una tarea se relaciona en el equipo, iv) de espacios de trabajo, relacionado a la actividad de los 
demás miembros del equipo y que han hecho y donde, v) de construcción del conocimiento individual, con información de la propia actividad, la cual contempla la utilidad de las aportaciones para resolución de problemas, el tiempo necesario y disponible, vi) de construcción del conocimiento de equipo, correspondiente a información utilizada para mantener al tanto a los miembros de equipo, vii) de actividad, acerca de cómo van las cosas, cambio de planes y modificación de roles, viii) de acción, especifica avances en las tareas, que sucede, que cambios, el nivel de actividad y frecuencia en el uso de un recurso, y ix) de situación, describe minuto a minuto los cambios en el sistema. Esta clasificación se enfoca en la información que comparte el equipo y la frecuencia en el uso de los recursos. Los tipos de awareness analizados se presentan en la Tabla 1.

Tabla 1. Tipos de awareness de acuerdo a [20,12].

\begin{tabular}{|l|l|}
\hline Awareness & Descripción \\
\hline De colaboración & $\begin{array}{l}\text { Se especializa en el trabajo centralizado o distribuido, síncrono o } \\
\text { asíncrono. }\end{array}$ \\
\hline De ubicación & $\begin{array}{l}\text { Representa información de la relación geográfica entre colaboradores y } \\
\text { su rango de atención. }\end{array}$ \\
\hline De contexto & Contempla eventos en un espacio virtual y los objetos que intervienen. \\
\hline Social & Proporciona información sobre roles y tareas que realizan los usuarios. \\
\hline De espacio de trabajo & Centrado en el tipo de actividades (paralelas o coordinadas). \\
\hline De situación & Enfocado a entender eventos, acciones, recursos y escenarios futuros. \\
\hline De tareas & Aporta información sobre cómo se completarán las tareas. \\
\hline De conceptos & Define cómo una actividad se relaciona con el conocimiento del equipo. \\
\hline $\begin{array}{l}\text { De construcción del co- } \\
\text { nocimiento individual }\end{array}$ & Comparte información de la propia actividad. \\
\hline $\begin{array}{l}\text { De construcción del co-- } \\
\text { nocimiento de equipo }\end{array}$ & $\begin{array}{l}\text { me utiliza con el fin de estar al tanto de los conocimientos de otros } \\
\text { miembros de equipo. }\end{array}$ \\
\hline De actividad & Aborda aspectos de planeación para la actividad y cambios que surgen. \\
\hline De acción & $\begin{array}{l}\text { Describe avances en las tareas, que sucede, que cambios, el nivel de } \\
\text { actividad y frecuencia en el uso de un recurso. }\end{array}$ \\
\hline
\end{tabular}

A partir de esta clasificación, se propone incluir awareness de tipo social y de tareas para ayudar al equipo en términos de organización y permitir al evaluador y los participantes formar una opinión más precisa de la actividad de los integrantes de equipo. Posteriormente, esto se puede implementar en diferentes escenarios colaborativos como realización de diagramas en el dominio de bases de datos relacionales, editores de texto y videojuegos. Para ayudar al equipo se podría utilizar agentes de manera similar a sistemas de tutores inteligentes $[21,22,23]$.

\section{Diseño de la aplicación}

\subsection{Análisis}

Retomando el problema de trabajar en ambientes tradicionales educativos, se necesita una aplicación donde un usuario pueda colaborar en equipo para 
construir diagramas de clases de manera colaborativa. El sistema debe permitir trabajar de manera fluida y organizada, además de aportar a los participantes y al evaluador una idea clara de la actividad de los usuarios en el equipo, esto para preparar a estudiantes de licenciatura en cursos de programación, particularmente diseño orientado a objetos.

Durante el trabajo de equipo asistido por computadoras, la comunicación y organización del equipo es mediante métodos directos (p. ej. chat, email, foro [6]), distrayendo en ocasiones a los participantes que realizan una tarea. Utilizar awareness en un Groupware permite mostrar información de manera indirecta al equipo y podría evitar el uso de un método directo, por ejemplo, para formar una opinión social durante el desarrollo de la actividad. Aprovechar la información proporcionada por awareness evita tener que escribir a otros integrantes de equipo. Hacer uso de un mecanismo de awareness puede evitar este tipo de retrasos permitiendo realizar una tarea en menor tiempo, facilitando de esta manera, la evaluación del producto construido colaborativamente y la participación del equipo por el uso de información de tipo social y de tareas.

La identificación de elementos necesarios para modelar aspectos de colaboración en la aplicación colaborativa, se realizó un experimento exploratorio donde se observa y analiza la actividad de estudiantes de licenciatura mientras resuelven ejercicios de diseño de software. Con esta observación se determinaron los elementos requeridos para modelar e identificar comportamientos individuales en un equipo de trabajo. Esto con la finalidad de obtener las dimensiones necesarias a incluir en un sistema Groupware de esta índole (p. ej. comunicación, cooperación, coordinación), particularmente una aplicación Groupware en diseño de software.

Para realizar este experimento exploratorio primero se indicaron las instrucciones del experimento a los usuarios (estudiantes). Después se presentó un problema de modelado de software. Se obtuvieron videograbaciones de estudiantes de licenciatura mientras trabajaban de manera colaborativa en actividades de diseño de software (ver Figura 3). Posteriormente se analizó el comportamiento de los usuarios con el objetivo de identificar aspectos que apoyan el trabajo de equipo.
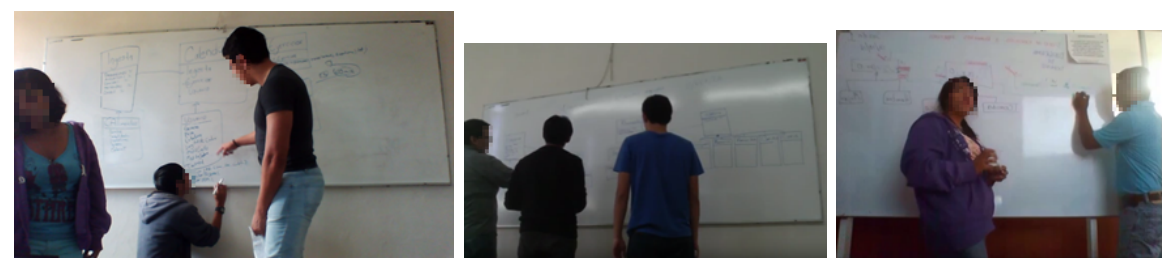

Fig. 3. Actividad colaborativa de diseño de software en un ambiente educativo.

Derivado de este análisis se propone una arquitectura funcional para la aplicación colaborativa de diseño de diagramas de clases y un prototipo que sirva como guía. 


\subsection{Arquitectura funcional}

Para implementar la aplicación de diseño de software se diseño una arquitectura funcional cliente-servidor basada en [24], [25], [26]. La Figura 4 muestra los componentes del cliente y del servidor de la aplicación.

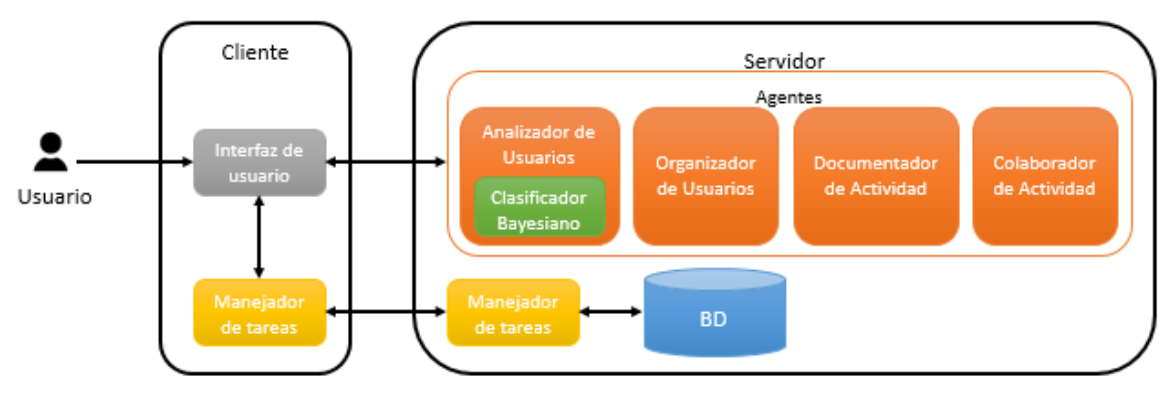

Fig. 4. Arquitectura de aplicación colaborativa de diseño de software para ambientes educativos.

A continuación, se describen los agentes propuestos que intervienen en el sistema:

- Analizador de Usuarios. Encargado de contabilizar las tareas de los usuarios durante la actividad de diseño de software, proporcionando información relacionada a la disponibilidad de usuarios para saber si están trabajando y cómo están trabajando. Esto con la finalidad de identificar comportamientos irregulares, por ejemplo, un usuario que construye y elimina clases para hacer creer a los compañeros de equipo que está trabajando, sin embargo, esa actividad pospone el cumplimiento de la meta que es construir el diagrama de clases completo. Entonces, el agente utiliza un historial de actividad para evaluar si el usuario está trabajando eficientemente. Además, clasifica a los usuarios de acuerdo a sus habilidades (construcción de clases, métodos, atributos, conectores), utiliza una escala de 1-5 donde 1 significa desempeño deficiente y 5 representa un desempeño competente. Para esta clasificación se podría utilizar un clasificador bayesiano que analice el historial de actividad de los usuarios.

- Organizador de Usuarios. Sugiere a los participantes de la actividad trabajar por subequipos según sus habilidades identificadas por el Agente Analizador de Usuarios, esto es, si el usuario ${ }_{1}$ es competente creando clases pero tiene habilidades deficientes en agregar atributos y el usuario $_{2}$ por el contrario es bueno agregando atributos pero es deficiente en habilidades para crear clases, el Agente Organizador de Usuarios sugerirá a estos usuarios apoyarse durante la actividad, estas decisiones las toma basado en el historial de actividad de los trabajos de los usuarios realizados anteriormente. 
Hacia el desarrollo de una aplicación colaborativa para el diseño de diagramas de clases ...

- Documentador de Actividad. Proporciona un informe de la actividad al evaluador, este informe contiene el desempeño individual de los usuarios durante la actividad y una sugerencia de cómo reorganizar los equipos de trabajo. Las sugerencias para reorganizar los equipos las ofrece basándose en la información recopilada por el Agente Analizador de Usuarios (habilidades demostradas por los usuarios).

- Colaborador de Actividad. Ayuda a los usuarios mediante el uso de sugerencias o notas en la construcción del diagrama de clases. El agente colabora de acuerdo al historial de trabajo del equipo y al contexto de la actividad que se realiza [27], por lo que se requeriría un entrenamiento previo con otros usuarios.

El lado del Cliente contiene la i) Interfaz de Usuario y el ii) Manejador de Tareas. La i) Interfaz de Usuario es la ventana donde los usuarios construyen los diagramas de clases y en el componente ii) Manejador de Tareas se procesan las tareas realizadas por los usuarios, por ejemplo, crear clase, atributo, método o conector. Por otra parte, el Servidor contiene, el i) Agente Analizador de Usuarios, ii) Agente Organizador de Usuarios, iii) Agente Documentador de Actividad, iv) Agente Colaborador de Actividad, v) Manejador de Tareas, y vi) una Base de Datos. El v) El Manejador de Tareas recibe las tareas procesadas en el lado del servidor y las envía a la Base de Datos. La Base de Datos procesa peticiones de tipo insert, delete, update y query.

Con la observación realizada en el experimento exploratorio, se identificó la importancia de proponer e incluir en la arquitectura agentes para la contabilización de tareas de los participantes en el equipo, para analizar y sugerir información para apoyar la coordinación de trabajo de equipo y para emitir reportes de actividad de las personas que colaboran en la actividad [28], [29].

\subsection{Interfaz de usuario cliente}

\section{Flujo de actividad}

Para utilizar la aplicación colaborativa se sigue el Flujo de Actividad que presenta la Figura 5. Seguir este Flujo permite al evaluador y a los participantes de equipo aprender sobre la actividad de cada integrante del equipo además de obtener sugerencias para mejorar la interacción del equipo en términos de coordinación y optimizar tiempos de trabajo.

La aplicación cuenta con un Login (ver Figura 6) para que se identifique el usuario. Posterior de iniciar sesión, se muestra una ventana para continuar trabajando en un diagrama de clases (ver Figura 7 a) ). Primero se selecciona el equipo al que pertenece el usuario y se muestran los archivos relacionados a el equipo seleccionado. Después, se selecciona un archivo para continuar trabajando en el diagrama de clases. Otra opción es crear un nuevo archivo (ver Figura 7 b) ) para trabajar en un diagrama de clases, se selecciona el equipo de trabajo, se escribe el nombre del archivo de diagrama de clases y se presiona el botón "Crear". 


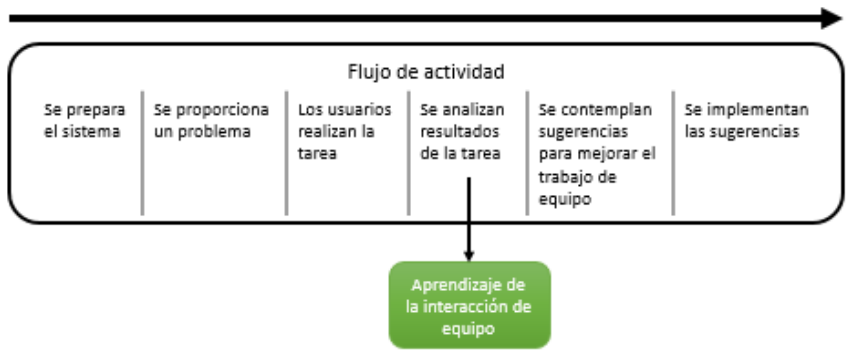

Fig. 5. Proceso para el uso de la aplicación colaborativa.

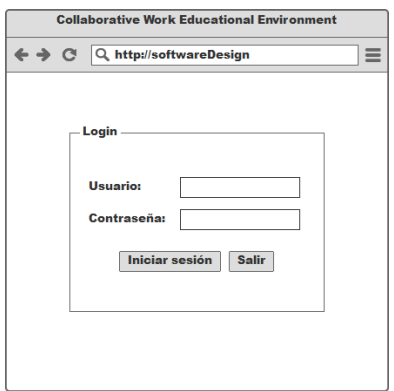

Fig. 6. Ventana para iniciar sesión en la aplicación de diseño de software.

Posteriormente de abrir un archivo existente o crear uno nuevo se muestra el entorno donde los usuarios colaboran en equipo para diseñar un diagrama de clases (ver Figura 8). En esta ventana los usuarios pueden agregar, modificar o eliminar una clase, un atributo, un método o un conector entre clases para construir un diagrama de clases. Además, pueden ver los usuarios que se encuentran colaborando en la actividad, y las tareas que cada uno realiza.

Un ejemplo de sugerencia de colaboración del Agente Organizador de Usuarios se muestra en el rectángulo 1) de la Figura 8, en esta sugerencia el Agente Organizador de Usuarios invita al usuario $o_{2}$ a ayudar al usuario ${ }_{1}$.

Por último, la Figura 9 presenta un informe generado por el Agente Documentador de Actividad. Este informe tiene el objetivo de ayudar al evaluador a comprender como se han comportado los usuarios durante la actividad, utilizando una escala de 1-5 donde 1 significa desempeño deficiente y 5 representa un desempeño competente.

Para generar este informe, los agentes utilizarían información de las tareas realizadas en comparación con los participantes en el equipo. Apoyando de esta manera el awareness de tipo Social. 
Hacia el desarrollo de una aplicación colaborativa para el diseño de diagramas de clases ...

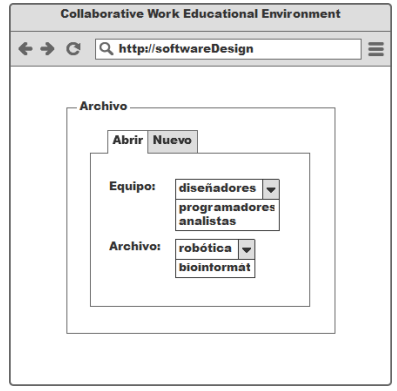

(a) Abrir archivo

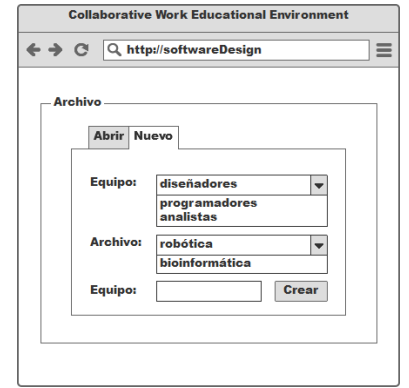

(b) Nuevo archivo

Fig. 7. a) Ventana para continuar trabajando en un diagrama de clases. b) Ventana para trabajar en un nuevo diagrama de clases.

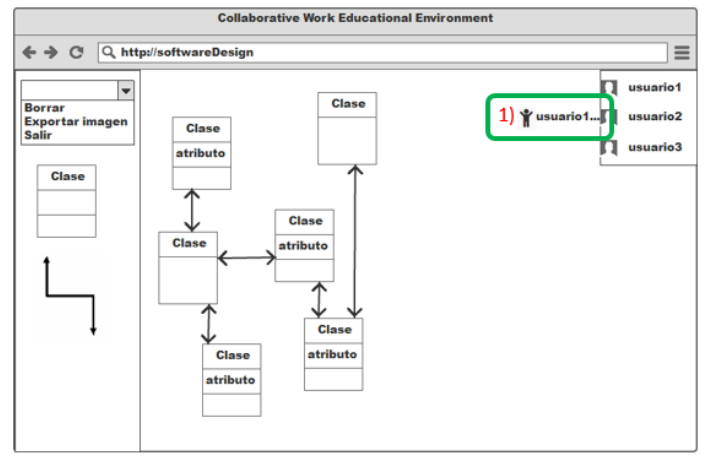

Fig. 8. Ventana para construir diagramas de clases de manera colaborativa en ambiente educativo.

\section{Conclusiones y trabajo futuro}

En este trabajo, se ha presentado un diseño inicial de una aplicación colaborativa de diseño de software en un ambiente educativo de manera conceptual. La finalidad de la aplicación es facilitar a los usuarios y a la persona que evalúa la actividad identificar como colaboran en equipo los usuarios cuando realizan una tarea. Con la arquitectura propuesta será posible llevar un registro de las tareas y acciones realizadas por cada integrante de equipo.

En etapas futuras de este trabajo, se espera finalizar la implementación de la arquitectura y aplicación propuesta en este artículo. Con la finalidad de incluir un mecanismo que mida el desempeño y contribución de los usuarios en relación a las actividades que desarrollan, esta medida es conocida como presencia social [3]. Con esta medición, se incluirán agentes, un agente que presente la presencia social de acuerdo al estilo de trabajo de los participantes (mediante una gráfica o conjunto de objetos/símbolos relacionados al contexto de la actividad). Este 


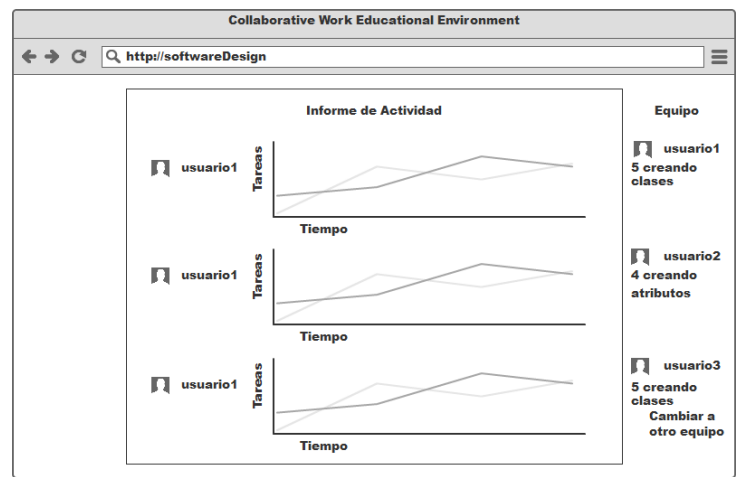

Fig. 9. Informe que muestra el historial de tareas en el tiempo durante la actividad.

agente podría ser implementado con la finalidad de proporcionar información que facilite a los usuarios completar la actividad en menor tiempo. Finalmente, incorporar otro agente para aportar sugerencias sobre como presentar la presencia social (ubicación de la información, tiempo para mostrar la información y cantidad de información a mostrar). Por lo tanto, la perspectiva final de nuestro trabajo es ofrecer información que facilite la actividad colaborativa y que ayude a formar una opinión social en los integrantes de equipo y el evaluador.

Agradecimientos. Los autores agradecen a los revisores de este documento sus útiles comentarios. El primer autor agradece al Consejo Nacional de Ciencia y Tecnología (CONACYT) por la beca otorgada para realizar estudios de posgrado (No. de beca: 413500).

\section{Referencias}

1. Palmer, T.D., Fields, N.A.: Computer supported cooperative work. Computer, 27 (5):15-17 (1994)

2. Galegher, J., Kraut, R.E., Egido, C.: Intellectual teamwork: Social and technological foundations of cooperative work. Psychology Press (2014)

3. Montané-Jiménez, L. G., Benítez-Guerrero, E., Mezura-Godoy, C., Pino, J. A.: Measuring Social Presence in Groupware Systems. In: Proceedings of the International Conference on CSCW in Design (CSCWD'2015), Calabria, pp. 200-205 (2015)

4. Presentación de la Monografía: Técnicas de la inteligencia artificial aplicadas a la educación. IA, Revista Iberoamericana de Inteligencia Artificial, (33):7-12 (2007)

5. Flores Quisbert, E.: Agentes inteligentes: El siguiente paso en la inteligencia artificial. Revista de Información, Tecnología y Sociedad, pp. 106 (2008)

6. Costaguta, R.: Una revisión de desarrollos inteligentes para aprendizaje colaborativo soportado por computadora. Revista Ingeniería Informática, 13:2013-2015 (2006) 
Hacia el desarrollo de una aplicación colaborativa para el diseño de diagramas de clases ...

7. Chaffey, D.: Groupware, workflow, and intranets: reengineering the enterprise with collaborative software. Gulf Professional Publishing (1998)

8. Ellis, C.A., Gibbs, S.J., Rein, G.: Groupware: some issues and experiences. Communications of the ACM, 34(1):39-58 (1991)

9. Xiao, W.P., Chi, C.Y., Yang, M.: On-line collaborative software development via wiki. In: Proceedings of the 2007 international symposium on Wikis, pp. 177-183, ACM (2007)

10. AssaultCube: What is assaultcube? http://assault.cubers.net/ (2013)

11. Mangano, N., LaToza, T.D., Petre, M., van der-Hoek, A.: Supporting informal design with interactive whiteboards. In: Proceedings of the SIGCHI Conference on Human Factors in Computing Systems, pp. 331-340, ACM (2014)

12. Herrera, A., Rodríguez, D., García-Martínez, R.: Taxonomía de mecanismos de awareness. In: XVIII Congreso Argentino de Ciencias de la Computación (2013)

13. Belkadi, F., Bonjour, E., Camargo, M., Troussier, N., Eynard, B.: A situation model to support awareness in collaborative design. International Journal of HumanComputer Studies, 71(1):110-129 (2013)

14. Bibbó, L. M.: Modelado de sistemas colaborativos. PhD thesis, Facultad de Informática (2009)

15. Galdámez, N., Sanz, C.V., De-Giusti, A. E.: Diseño de un entorno web colaborativo orientado al ámbito educativo para desarrollar la técnica de brainstorming. In: XVII Congreso Argentino de Ciencias de la Computación (2011)

16. Robert, L. McLeod, A., Davis, A. R. Erp configuration: Does situation awareness impact team performance? In: System Sciences (HICSS), 2011 44th Hawaii International Conference on, pp. 1-8, IEEE (2011)

17. Epic Games: Gears of War. https://gearsofwar.com/ (2006)

18. Wendel, V., Gutjahr, M., Göbel, S., Steinmetz, R.: Designing collaborative multiplayer serious games. Education and Information Technologies, 18(2):287-308 (2013)

19. Gumienny, R., Gericke, L., Wenzel, M., Meinel, C.: Supporting creative collaboration in globally distributed companies. In: Proceedings of the 2013 conference on CSCW, pp. 995-1007, ACM (2013)

20. Antunes, P. Herskovic, V., Ochoa, S. F., Pino, J. A.: Reviewing the quality of awareness support in collaborative applications. Journal of Systems and Software, 89:146-169 (2014)

21. Cataldi, Z., Salgueiro, F.A., Lage, F.J., Martínez, R. G.: Sistemas tutores inteligentes: los estilos del estudiante para selección del tutorizado. In: VII Workshop de Investigadores en Ciencias de la Computación (2005)

22. Peña, C. I., Marzo, J. L., De la Rosa, J. L., Fabregat, R.: Un sistema de tutoría inteligente adaptativo considerando estilos de aprendizaje. Revista UIS ingenierías, 1(2) (2012)

23. Cataldi, Z., Lage, F. J.: Modelado del estudiante en sistemas tutores inteligentes. TE \& ET (2010)

24. Benítez-Guerrero, E., Mezura-Godoy, C., Montané-Jiménez, L. G.: Context-aware mobile collaborative systems: Conceptual modeling and case study. Sensors, 12 (10):13491-13507 (2012)

25. Carrascosa, C., Julián, V., Soler, J.: Una arquitectura de sistema multi-agente para la recuperación y presentación de la información. In: La representación y la organización del conocimiento en sus distintas perspectivas: su influencia en la recuperación de la información: actas del IV Congreso ISKO-España EOCONSID’99, 22-24 de abril de 1999, Granada, pp. 291-296, Universidad de Granada (1999) 
26. Gil, A., García, F.: Un sistema multiagente de recuperación de objetos de aprendizaje con atributos de contexto. In: Workshop on Agents and Multi-agent Systems for Enterprise Integration (ZOCO 2007) at CAEPIA, pp. 1-11 (2007)

27. Baylor, A. L., Kim, Y.: Simulating instructional roles through pedagogical agents. International Journal of Artificial Intelligence in Education, 15(2):95-115 (2005)

28. Hurtatiz, Y. E. M., Rengifo, Y. S. P., Rojas, E. E. M.: Sistemas tutores inteligentes como apoyo en el proceso de aprendizaje. Redes de Ingeniería, 6(1):25-44 (2015)

29. Hernández, J. A. D., Rengifo, Y. S. P.: Los sistemas tutores inteligentes y su aplicabilidad en la educación. Revista horizontes pedagógicos, 17(2):104-116 (2016) 